\title{
Structural Health Monitoring (SHM) of Machine Using a Combination of Non-Destructive Testing for Heavy Manufacturing Industry
}

\author{
Ashish Khaira ${ }^{1, *}$, Ravi.K. Dwivedi ${ }^{2}$, and Sanjay Jain ${ }^{1}$ \\ ${ }^{1}$ Department of Mechanical Engineering, SATI, Vidisha, India \\ ${ }^{2}$ Department of Mechanical Engineering, MANIT, Bhopal, India
}

\begin{abstract}
Markets are affected by assorted consumer requirements, which insist on superior quality, shorter delivery time, better customer support, and lower prices. Simultaneously, product life cycles are becoming shorter. Success relies on having either a cost-benefit or a value benefit, or, both in any competitive context. Therefore, non-destructive techniques (NDT) become vital but in the conventional system, the maintenance personnel has to visit the machine that consumes time and energy. In the present COVID-19 situation and to save energy and time, there is a necessity of making condition monitoring contactless as much as possible. Therefore, in this research work, a structural health monitoring analysis presented that covers: firstly, enlisting of the NDT infrastructure commonly available in heavy manufacturing industries; secondly, common causes and reasons of machine failures and finally, discusses need of embedded structural health monitoring (e-SHM) system with the combination of NDT in place of existing monitoring practice. The presented work suggested that a combination of NDT with e-SHM is better for timely fault detection to ensure effective condition monitoring.
\end{abstract}

\section{Introduction}

Generally, excessive repairs results [1] in the reduction of performance, efficiency, and useful operating life. It is productive [2] to conduct fault diagnosis at early stages. It is essential for higher reliability and lower failure rate. Non-destructive testing (NDT) involves surface or subsurface inspection for flaws without destructing it. NDTs are efficient methods [3]-[6] that help in reducing Mean Time to Repair (MTTR) by enhancing troubleshooting and increasing Mean Time between Failure (MTBF) due to pro-activeness.

Various industries apply NDT methods for fault detection and its history starts from the 1800s. There are various events in history [7] of NDT techniques, key events are shown in figure 1.Various researchers [8]-[10] showed that the non-destructive testing techniques have several advantages, while there are some disadvantages too. Therefore, a single NDT technique cannot give highly reliable fault detection. To achieve this objective, it is necessary to combine multiple NDT techniques.

The research work in ref. [11], showed the use of multiple NDTs on additively manufactured metallic specimens that has an e-SHM system. The author concluded that the e-SHM system with NDT combination could be helpful in fault detection at the early stages.

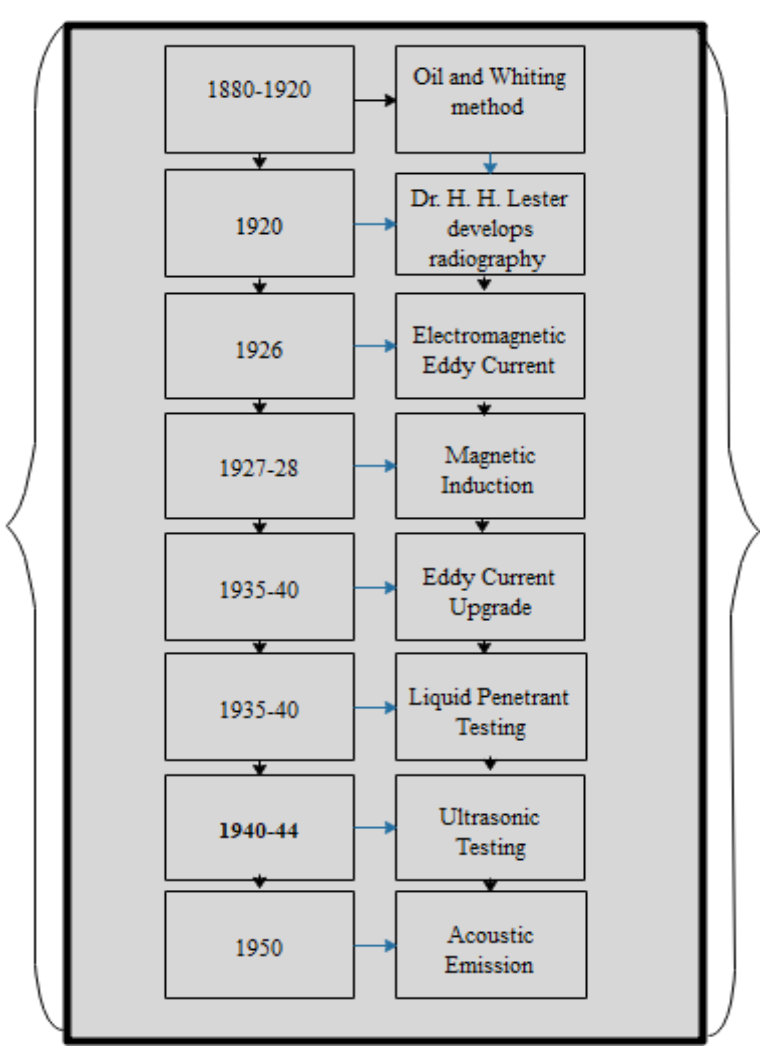

Fig. 1. Historical Events Related to NDT

\footnotetext{
* Corresponding author: ashish_2285@yahoo.co.in
} 


\section{Condition Monitoring Using NDT}

Non-destructive testing techniques play a important role in industrial condition monitoring of machines. The current section and upcoming subsection covered NDT equipment, maintenance practices, etc.

\subsection{Condition Monitoring Equipment's}

This sub-section enlists the NDT infrastructure commonly available in heavy manufacturing industries, which are as follows:

$\checkmark$ Sound Sensor (see figure 2)

$\checkmark$ Dynamic Balancer (see figure 3)

$\checkmark$ Accurate Oil Care (For moisture removal from oil)

$\checkmark$ Patch Test

$\checkmark$ Laser Shaft Alignment (see figure 4)

$\checkmark \mathrm{M} / \mathrm{c}$ Condition Tester

$\checkmark$ Shock Pulse Wave Tester

$\checkmark$ Sound Pressure Meter (For checking sound in blower)

$\checkmark$ Tachometer

$\checkmark$ Ultrasonic and Eddy Current

$\checkmark$ Thermography (see figure 5)

$\checkmark$ Vibration Tester (see figure 6)

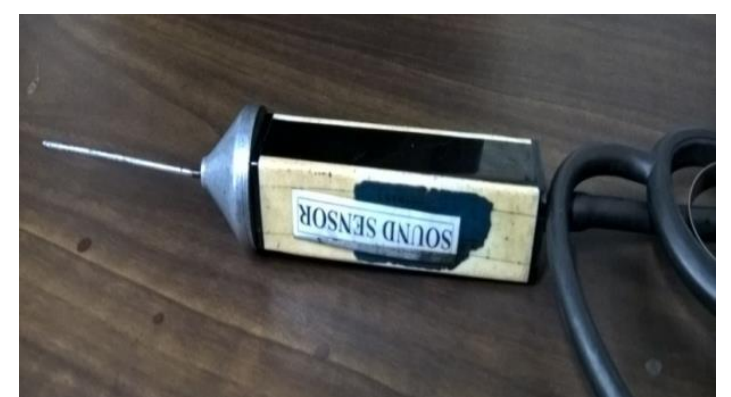

Fig. 2. Sound Sensor

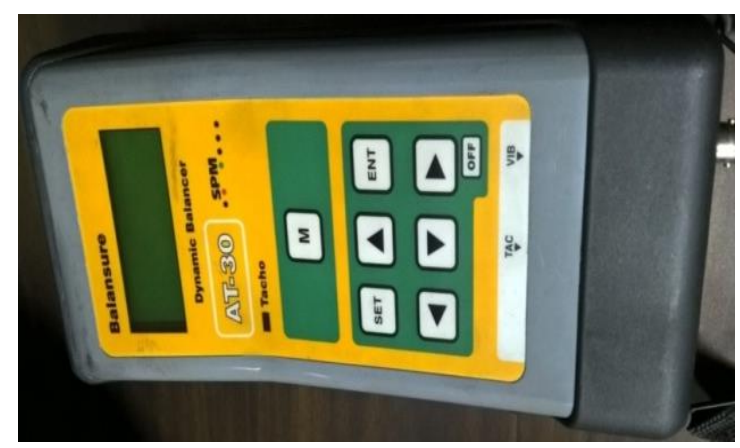

Fig. 3. Dynamic Balancer

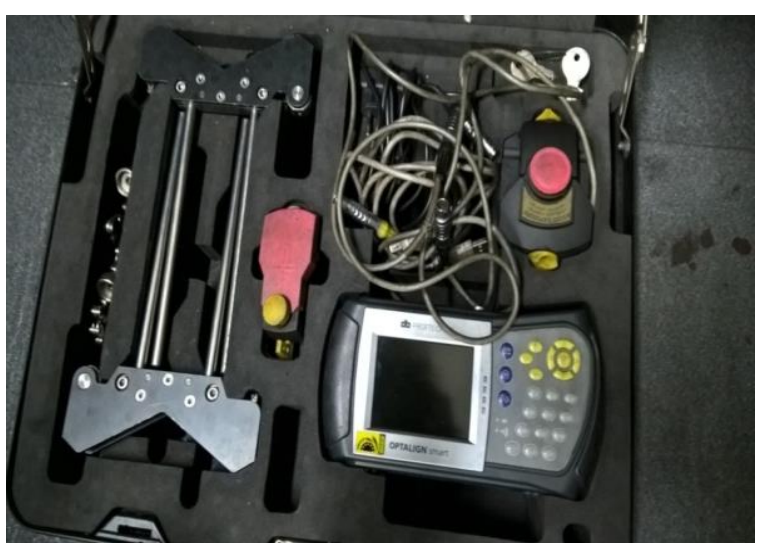

Fig. 4. Laser Shaft Alignment

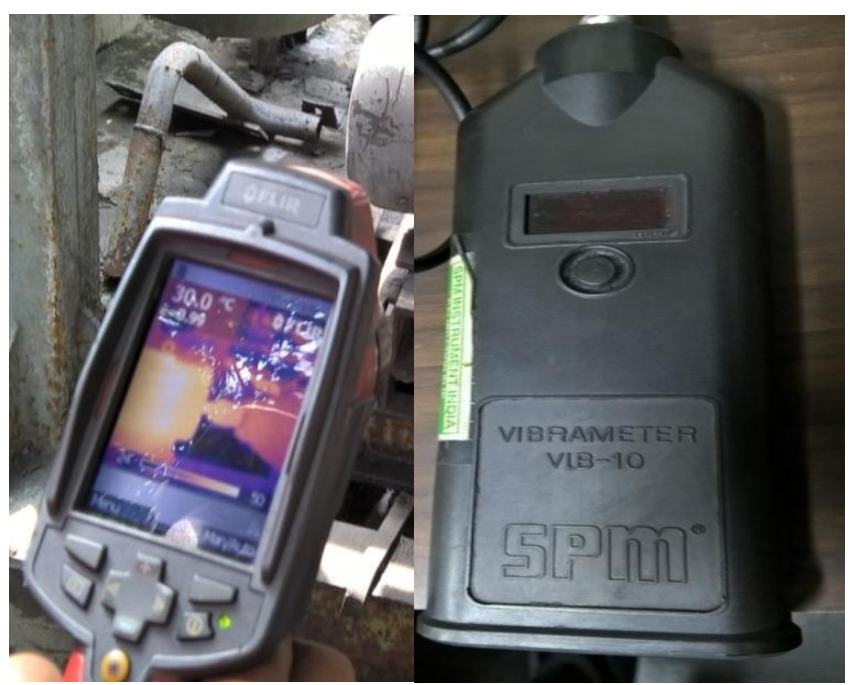

Fig. 5. Thermography

Fig. 6. Vibration Tester

\subsection{Maintenance Approach for Machine}

The machine has separator which separates oil from compressed air. The filter (see figure 7) and separator changes after $3000 \mathrm{Hr}$. of working instead of cleaning and reusing in this heavy industry. The age of air filter is low because of carbon dust so they are cleaned one or two times after that it changed.

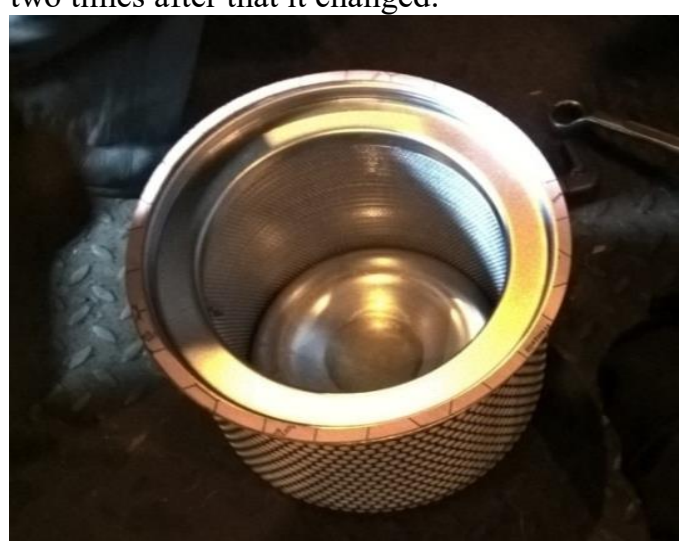

Fig. 7. Oil Filter

\section{Result \& Discussions}

\subsection{Common Failure \& Their Causes}


- Scaling in the heat exchanger, Due to this cooling was improper. So the machine heated up.

- Sometimes due to improper viscosity of lube oil

- Sensors problems.

\subsection{Suggestion's for Improvement}

After analyzing the maintenance approach, some recommendations based on observation to enhance maintenance performance also provided, which are as follows -

\section{(1). Vibrations Testing-}

- As per ISO 10816-3:2009 (see table 1) for a machine having motor of $90 \mathrm{KW}$ with the flexible foundation the vibration limits in $\mathrm{mm} / \mathrm{sec}$ are-

Table 1. Range of Vibration Values

\begin{tabular}{|c|c|c|c|}
\hline Location & Green & Yellow & Red \\
\hline Motor & $2.3-4.5$ & $4.5-7.1$ & $>7.1$ \\
\hline Gear Box & $2.3-4.5$ & $4.5-7.1$ & $>7.1$ \\
\hline Compressor & $2.3-4.5$ & $4.5-7.1$ & $>7.1$ \\
\hline
\end{tabular}

- Shock pulse vibration measurement of the gearbox is recommended once a month.

- Prevention of False Brinelling- Turn the shafts at least once per week. Many spare elements are damaged just after a few weeks of operation just because of the false brinelling effect.

- Check the amps of the motor as it is one of the best measures for bearing defect detection \& prevention

\section{(2). Lubrication-}

- Analysis of following parameter- Viscosity, Moisture, Wear Particle

- Drive motor-

$\checkmark$ The bearing at the non-drive end side is greased for life.

$\checkmark$ The bearing at the drive end side is lubricated with the oil system, so frequent check of oil level is recommended.

\section{(3). Ultrasonic Testing and Eddy Current-}

- Once a month for motor, gearbox \& compressor.

(4). e-SHM System - In present COVID-19 situation and to save energy and time, there is a necessity of making condition monitoring contactless as much as possible. Therefore there is a need for embedded structural health monitoring (e-SHM) system with a combination of NDT in place of existing monitoring practice. The central idea of the e-SHM system is embedded capillaries in the interior of a machine component. A sudden change in pressure in the capillary gives the crack indication. The implementation of such a system needs a proper feasibility study and cost analysis.

\section{Conclusions}

It was already highlighted that in the conventional condition monitoring system, the maintenance personnel has to visit the machine that consumes time and energy.
In the present COVID-19 situation and to save energy and time, there is a necessity of making condition monitoring contactless as much as possible. This research work showed the NDT infrastructure commonly available in heavy manufacturing industries, discussed common causes and reasons of failure, and discussed the need for embedded structural health monitoring (e-SHM) system with a combination of NDT in place of existing monitoring practice. Presented work suggested that a combination of NDT with e-SHM is better for timely fault detection to ensure effective condition monitoring. Furthermore, the effectiveness of such type of combination of condition monitoring needs analysis of improvement in reliability, reduction in failure rate, and economic aspects.

We are thankful to the SATI-Vidisha for providing the required facilities and permissions needed for the successful completion of this work; World Bank, TEQIPIII, and NPIU for providing the necessary support and funds.

\section{References}

1. Escaler Xavier, Egusquiza Eduard, Farhat Mohamed, Avellan Franc-ois, Coussirat Miguel, Mechanical Systems and Signal Processing, Detection of cavitations in hydraulic turbines, 20, 983-1007 (2011).

2. Garnier Christian, Pastor Marie-Laetitia, Florent Eyma, Lorrain Bernard, Composite Structures, The detection of aeronautical defects in situ on composite structures using Non Destructive Testing, 93, 13281336 (2011).

3. Wilcox Mark, Downes George, A Brief Description of NDT Techniques (Insight NDT Equipment Limited, England, 2003).

4. D’Orazioa T., Leoa M, NDT\&E International, Ultrasonic Testing for Flaw Detection in Mild Steel Sample, (2008).

5. Krautkramer, J., H. Krautkramer, Ultrasonic Testing of Materials (Springer Publications, New York, 1990).

6. Khaira, Ashish, and Ravi K. Dwivedi, A State of Art Critique Review of Role of Optimization in NDTS for Effective Condition Monitoring (Advanced Condition Monitoring and Fault Diagnosis of Electric Machines, IGI Global, 2019).

7. Chuck Hellier, Handbook of Nondestructive Evaluation (McGraw-Hill Companies, 2003).

8. Lim Malcolm K., Cao Honggang, Construction and Building Materials, Combining multiple NDT methods to improve testing effectiveness, 38, 13101315 (2013).

9. Khangar V. S., Jaju S. B., International Journal of Emerging Technology and Advanced Engineering, A Review of Various Methodologies Used for Shaft Failure Analysis, 2, (2012).

10. Verma Ashutosh, Srivastava Shakun, International Journal of Enhanced Research in Science Technology \& Engineering, Review on Condition Monitoring Techniques Oil Analysis, Thermography and Vibration Analysis, 7, 18-25 (2014). 
11. Strantza, M., Aggelis, D. G., De Baere, D., Guillaume, P., \& Van Hemelrijck, D. Sensors, Evaluation of SHM system produced by additive manufacturing via acoustic emission and other NDT methods. , 15, 10(2015). 